\title{
Importancia de los deslizamientos en el Parque Nacional Podocarpus, Loja, Ecuador
}

\author{
Importance of landslides in Podocarpus National Park, Loja, Ecuador \\ Pablo Lozano1 y Rainer Bussmann²
}

Publicación: 08/12/2005

\section{Resumen}

Se presentan datos preliminares de la flora pionera en deslizamientos naturales y antrópicos en el Parque Nacional de Podocarpus (PNP), así como de la vegetación antes de estos eventos. Los deslizamientos naturales fueron seleccionados y distribuidos entre los 2100 a $3200 \mathrm{~m}$ de altitud, (216 parcelas), mientras que los deslizamientos antropogénicos fueron estudiados alrededor de la zona de amortiguamiento, entre los 1900 a 2800 m (216 parcelas). El muestreo se efectuó según Blanquet (1979) tomando como referencia la gradiente altitudinal. Los deslizamientos naturales y antrópicos fueron seleccionados en dos sitios hacia el flanco oriental (144 parcelas) y cuatro sitios en el flanco occidental (288) parcelas, totalizando 432 parcelas. Para la vegetación natural, el tamaño de la parcela fue establecido de acuerdo al tipo de vegetación (herbácea, arbustiva y arbórea). El bosque natural presenta 75 familias, 185 géneros y 412 especies, de estas 58 son endémicas entre los 2800 m y 3200 m; deslizamientos naturales con 56 familias, 127 géneros y 264 especies; y deslizamientos antropogénicos con 69 familias, 127 géneros y 313 especies. No existen diferencias entre la vegetación pionera de los deslizamientos naturales y antrópicos, sin embargo la gradiente influencia directamente en el tipo de vegetación y estructura. Siendo el estrato herbáceo bajo el más representativo, luego el estrato herbáceo de tamaño mediano y finalmente el estrato arbustivo, especialmente en el rango 2400 a 2700 $\mathrm{m}$. Se registra que en los dos flancos del PNP existe una elevada frecuencia de deslizamientos principalmente entre los 2200-2500 m. Existen algunos grupos predominantes o que restablecen estas áreas, entre ellas las Criptógamas, los estratos herbáceos con familias dominantes (Violaceae, Cyperaceae, Poaceae, Blechnaceae, Lycopodiaceae), el estrato arbustivo con otro juego de familias (Asteraceae, Melastomataceae, Ericaceae, Poaceae, Aquifoliaceae), y en el bosque todavia otras familias dominantes (Asteraceae, Clusiaceae, Bromeliaceae, Ericaceae, Lycopodiaceae, Melastomataceae, Orchidaceae, Poaceae, Cunoniaceae).

Palabras clave: Bosque montano, deslizamientos, vegetación pionera, Parque Nacional Podocarpus, Ecuador

\begin{abstract}
We present preliminary data on the pioneer vegetation found on natural and man-made landslides in the Parque Nacional de Podocarpus (PNP). The natural landslides selected were distributed between 2100 and $3200 \mathrm{~m}$ (216 study sites), while the man-made landslides were situated between 1900 and $2800 \mathrm{~m}$ above sealevel (216 study sites). Sampling followed Blanquet (1979) and the altitudinal gradient was used as reference. The land-slides were selected in two areas on the eastern flank (144 study sites) and in four areas on the western flank ( 288 study sites), totaling 432 study sites. For the natural vegetation the size of the study sites was selected according to vegetation type (herbaceous, shrubby, arboreal). The natural forest has 75 plant families, 185 genera and 412 species, 58 of the latter are endemic to the region between $2800 \mathrm{~m}$ and $3200 \mathrm{~m}$ above sealevel; natural land-slides have 56 families, 127 genera and 264 species; and man-made land-slides have 69 plant families, 127 genera and 313 species. There are no differences between the vegetation of the natural and the man-made land-slides, but rather the gradient has a direct influence on vegetation type and structure. The low herbaceous layer is the dominant feature, followed by medium-sized herbaceous plants and finally the shrub layer, especially between 2400 and $2700 \mathrm{~m}$ above sea-level. Within the PNP there are an elevated number of natural land-slides especially between 2200 and $2500 \mathrm{~m}$ above sea-level. There are some plant groups which predominate in these areas, such as Cryptogams, the herbaceous layer has some dominant plant families (Violaceae, Cyperaceae, Poaceae, Blechnaceae, Lycopodiaceae), as has the shrub layer (Asteraceae, Melastomataceae, Ericaceae, Poaceae, Aquifoliaceae), while in the natural forest other families are dominant (Asteraceae, Clusiaceae, Bromeliaceae, Ericaceae, Lycopodiaceae, Melastomataceae, Orchidaceae, Poaceae, Cunoniaceae).
\end{abstract}

Keywords: Montane forest, landslides, pioneer vegetation, Parque Nacional Podocarpus, Ecuador

${ }^{1}$ University of Hohenheim, Institute of Botany and Botanical Garden, Garbenstr.30, D-70599 Stuttgart, Germany

${ }^{2}$ University of Hawaii, Harold L. Lyon Arboretum, 3860 Manoa Rd., Honolulu, HI 96822,U.S.A. 


\section{Introducción}

A través de varios años, la recuperación de la vegetación ha sido estudiada como un importante rol en la ecología de plantas (Peet \& Christensen, 1980). La perturbación del ambiente tiene una larga tradición en investigación en diferentes tópicos así como restauración y sucesión (White \& Jentsch, 2001). Algunos estudios sugieren que los procesos de perturbación natural juegan un importante rol para mantener la biodiversidad (Christensen et al., 1989), estos procesos de regeneración y sucesión son importantes desde el punto de vista de la renovación del bosque por el cambio causado por actividades antropogénicas o naturales. Sin embargo estos procesos de fragmentación del hábitat pueden también incrementar las tasas de perturbación (Bergeron \& Brisson, 1990).

Los deslizamientos naturales y antropogénicos son comunes en los Andes del Ecuador, produciendo serios y continuos daños (Benítez, 1989), desafortunadamente solo pocos estudios se han realizado en este país (Ohl \& Bussmann, 2004; Stern, 1992; Benítez, 1989).
Estos deslizamientos han sido influenciados por una serie de fenómenos internos (termoplásticos) $\mathrm{y}$ factores ambientales externos, principalmente el clima y otros como pendiente, tipo de suelo, movimientos sísmicos; que algunas veces combinados con construcción de caminos, son la principal actividad para producir movimientos de tierra y deslizamientos.

Los Andes han sido influenciados por una serie de drásticos procesos climáticos con influencia directa de movimientos de tierra y conformación de ecosistemas óptimos para repoblación de algunos tipos de plantas, como parte de una dinámica natural. Los regímenes de perturbación tienen implicaciones críticas como los efectos de borde, la ruptura de interacción planta-animal y la cadena que esta conlleva, entre otros tópicos ecológicos como pérdida de especies e invasión de especies externas, las cuales están inmersas y juegan un importante rol en los procesos naturales de recuperación de la vegetación después de los deslizamientos.

El presente estudio tiene como objetivos, caracterizar la vegetación pionera en los deslizamientos naturales y antrópicos del Par-

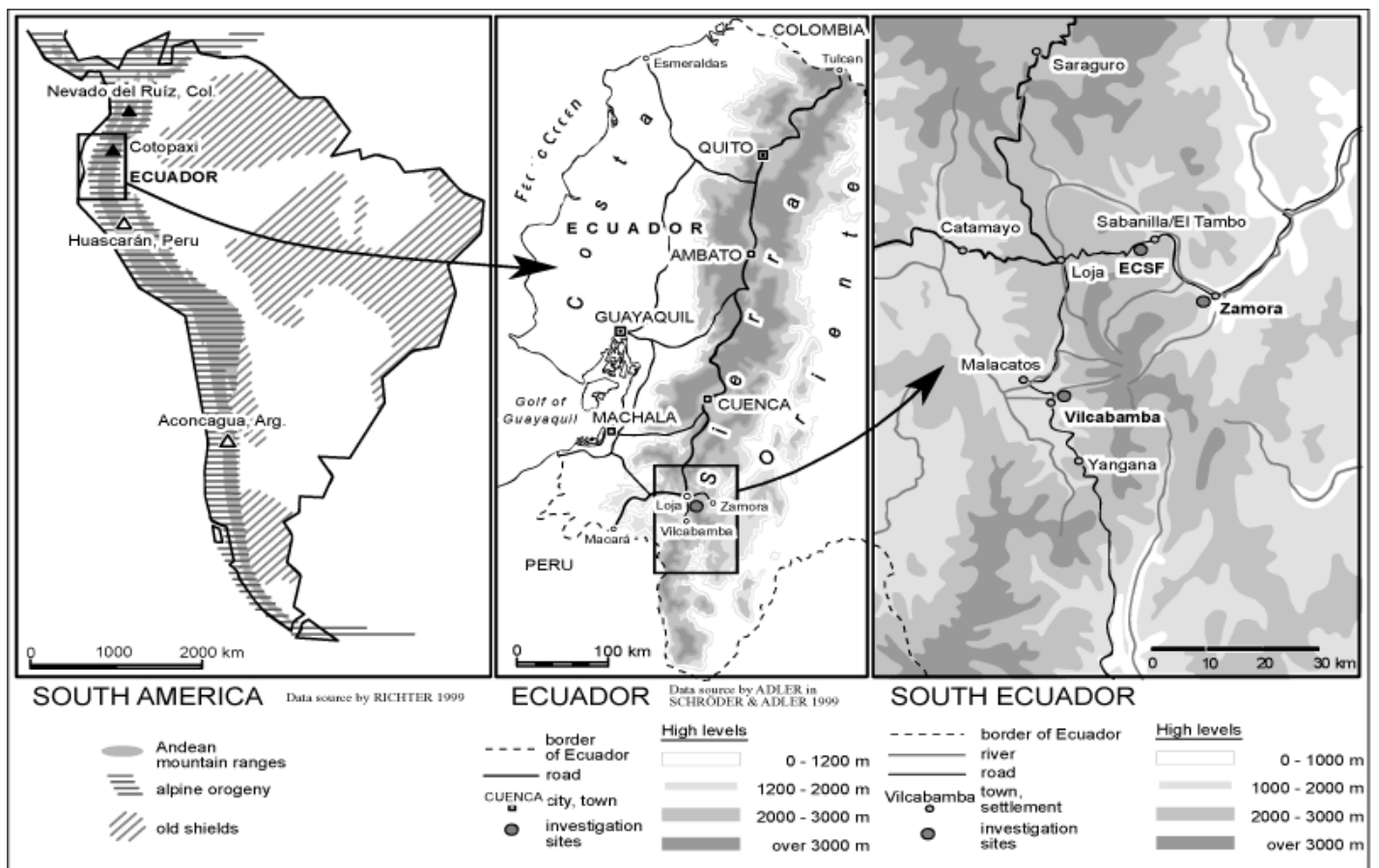

Figura 1. Ubicación del área de estudio. 
que Nacional Podocarpus, analizar la regeneración y cambio estructural según la gradiente altitudinal tanto en deslizamientos naturales como en antrópicos y reconocer la vegetación natural junto a los deslizamientos.

\section{Área de Estudio}

El Parque Nacional Podocarpus (PNP) creado en 1982, se ubica al sur del Ecuador en $\operatorname{los} 4^{\circ} 10^{\prime} \mathrm{S}, 7^{\circ} 04^{\prime} \mathrm{O}$ (Apolo, 1984), con una superficie de 146280 ha, y un rango altitudinal que varia entre 1000 y 3600 m (Fig. 1).

Existen dos denominaciones para la vegetación de esta área:

\section{Según Cañadas (1983)}

bosque muy húmedo premontano (bmh--PM) bosque muy húmedo montano bajo (bmh-MB) bosque pluvial montano (bp-M) bosque muy húmedo montano (bmh-M) bosque húmedo montano bajo (bh-MB)

\section{Según Madsen (1989)}

Bosque lluvioso-montano (1000-1500 m) Bosque Montano Oriental (1500-3000 m ) Páramo y Chaparro (3000 y 3700 m)

Bosque Montano Occidental entre (2500-3000 m)

Adicionalmente existe un tratamiento fitosociológico de la vegetación en la reserva San Francisco, al limite norte del PNP (Bussmann, 2002). Aquí se describen cuatro tipos de bosques, subdivididos en varios estratos. Así mismo, Quizhpe et al. (2002) identifica seis comunidades vegetales en los páramos del Parque Podocarpus.

Datos existentes señalan que la composición de los bosques nublados y páramos del sur, son muy particulares y diferentes a las formaciones del resto del país (Herbario, 2000). Un alto epifitismo fue registrado en el sector de San Francisco con 627 especies (Bussmann, 2001). En los bosques montanos de Cajanuma a $2800 \mathrm{~m}$, se encontraron 70 especies de árboles (Madsen com. pers.), mientras que en los páramos sobre los 2800 se encontraron 135 especies (Keating, 1995). Otro estudio de los páramos reconocen 221 especies en 93 géneros y 61 familias, identificándose 34 especies endémicas que ocurren principalmente en las comunidades arbustivas (Quizhpe et al., 2002). Existen 211 especies endémicas en el PNP (Valencia et. al., 2000) con 99 especies exclusivas, situándolo como una de las áreas protegidas con el mayor endemismo a nivel nacional (Lozano et al. 2003). Esta síntesis florística nos sitúa en un área de alta biodiversidad.

Representa la región más baja en los Andes del país, la fisiografía esta influenciada principalmente por la cordillera oriental que cruza de norte a sur el PNP y perpendicular a esta se desprende la cordillera de Paredones que va de oeste a este (Keating, 1995). Varias filas de cordilleras menos pronunciadas

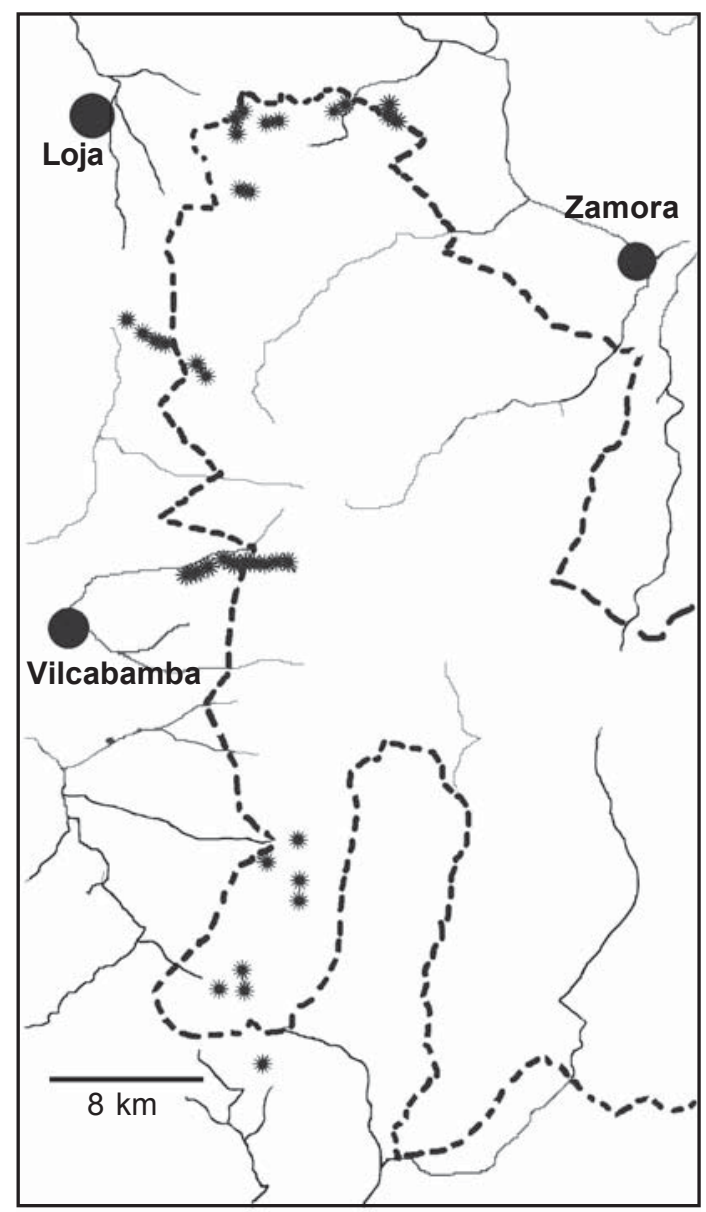

Figura 2. Ubicación de las parcelas y sitios de estudio en la parte occidental del Parque Nacional Podocarpus. 
se desprenden de la cordillera oriental formando microcuencas y valles hacia ambos lados este y oeste del parque.

El estudio de los deslizamientos naturales, antropogénicos y de vegetación natural se desarrolla generalmente en la parte occidental del PNP, de norte a sur desde; San Francisco (sector nor-oriental) entre un rango altitudinal de 2100 a $3000 \mathrm{~m}$. Sector El Tiro (norte del PNP), desde 2700 a 3200 m, avanzando por la cordillera oriental hacia Cajanuma y en este sector desde 2500 a 3200 m, en la parte alta de Vilcabamba sector (centro-sur), desde los 2600 a 3100 m, en Cerro Toledo sector (centro-sur), desde 2500 a $3400 \mathrm{~m}$, y en la Cordillera de Sabanilla sector (sur-oriental), entre 2300 a 2900 m (Fig. 2).

Para los deslizamientos antropogénicos se ha seleccionado el área de amortiguamiento del PNP, vía Loja-El Paso (Tiro)-Zamora entre $2500 \mathrm{~m}$ a los 2800 y descendiendo a $\operatorname{los} 1900 \mathrm{~m}$. En la vía Cajanuma-Centro de información del PNP entre 2500 a 2800 m, en la vía YanganaSabanilla y Valladolid desde los $2500 \mathrm{~m}$ ascendiendo a los 2800 y descendiendo a $1900 \mathrm{~m}$.

\section{Material y Métodos}

Se diseñó el muestreo de campo, tomando en cuenta el rango altitudinal y se ubicó las parcelas cada $100 \mathrm{~m}$. Siguiendo la metodología de Braun Blanquet (1979), se estableció parcelas de 1x5 m, subdividiéndolas en 1x1 m, en cada subcuadrante se registran los diferentes estratos herbáceo o arbustivo, tomando datos de porcentajes de presencia por especie, esto para deslizamientos naturales y antropogénicos.

En cada sitio seleccionado, se establecieron 72 parcelas (36 naturales y 36 antrópicas), de uno por cinco metros a diferentes altitudes $[(1800-2100 \mathrm{~m}) /(2200-2450 \mathrm{~m}) /($ sobre los 2500 $\mathrm{m})$ ]. Existen dos sitios hacia el flanco oriental (144 parcelas) y cuatro sitios al flanco occidental (288 parcelas), en total suman 432 parcelas.

Para la vegetación natural, se siguió la misma metodología de muestreo de Braun Blanquet (1979) y de acuerdo al tipo de vege- tación se establecieron parcelas de $5 \times 5 \mathrm{~m}$ en vegetación herbáceo y $10 \times 10 \mathrm{~m}$ en vegetación arbustiva y 10x50 m en vegetación arbórea, tomando los porcentajes de cobertura vegetal por especies.

En las parcelas se registraron los siguientes datos: posición geográfica, altitud, inclinación, y se registraron todas las especies herbáceas, arbustivas y arbóreas. Tomando en cuenta el número de parcela y fecha.

Se estableció 18 parcelas en vegetación natural junto a los deslizamientos naturales, tres por sitio e igualmente distribuidas en la gradiente, con la finalidad de comparar diversidad y composición florística previo el suceso de deslizamiento.

\section{Muestreo de Suelos}

En deslizamientos naturales y antrópicos, se tomaron tres muestras de suelos por sitio de norte a sur a $15 \mathrm{~cm}$ de profundidad, muestreos distribuidas en la gradiente, para posterior análisis de laboratorio en la Universidad Nacional de Loja, donde se analizó materia orgánica $(\mathrm{MO})$, acidez $(\mathrm{pH})$, nitrógeno disponible $(\mathrm{N})$ y textura.

Para el análisis de los suelos en vegetación natural se realizaron calicatas para muestreo de suelos, diferenciando los perfiles existentes, a una profundidad de $30-60 \mathrm{~cm}$, dependiendo del tipo de suelo. Adicionalmente se registró la temperatura del suelo, textura y estructura. Los muestreos fueron distribuidos igualmente en la gradiente altitudinal.

\section{Análisis de Herbarios}

Todo el material herborizado se identificó en el herbario LOJA de la Universidad Nacional de Loja y QCA de la Universidad Pontificia Católica de Quito y Museo de Ciencias Naturales del Ecuador respectivamente.

Se empleo literatura especializada, como es el Catálogo de las plantas vasculares del Ecuador (Jørgensen \& León-Yánez, 1999); algunos volúmenes de la serie Flora of Ecuador (Harling \& Andersson, 1986-2003); el Li- 


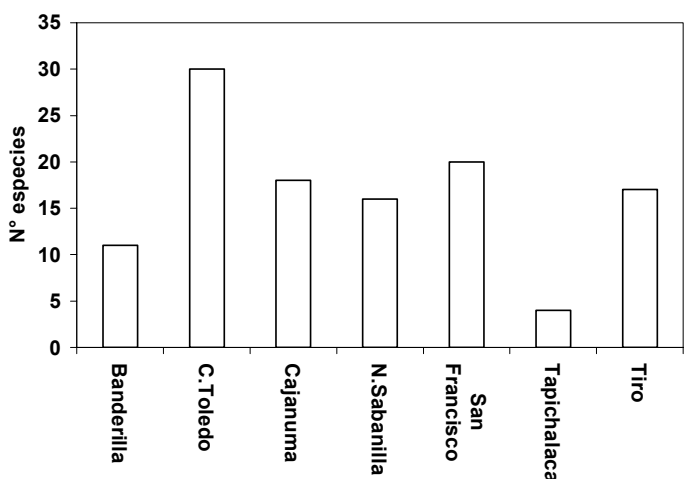

Figura 3. Número de especies endémicas encontradas por sector en vegetación natural.

bro rojo de las plantas endémicas de Ecuador (Valencia et al., 2000); así como el Catálogo de plantas vasculares del Perú (Brako y Zarucchi, 1993), entre otros.

\section{Resultados}

Los bosques montanos y páramos muestreados junto a los deslizamientos del Parque Podocarpus muestran una alta diversidad y especialmente endemismos. Se registraron 412 especies, de estas 58 son endémicas para el parque generalmente distribuidas entre $\operatorname{los} 2800$ a $3200 \mathrm{~m}$, en vegetación arbustiva de páramo «Ecotono». Cerro Toledo al sur del Parque destaca un alto endemismo (Fig. 3), variables ambientales en el ecotono, proveen características especiales para la

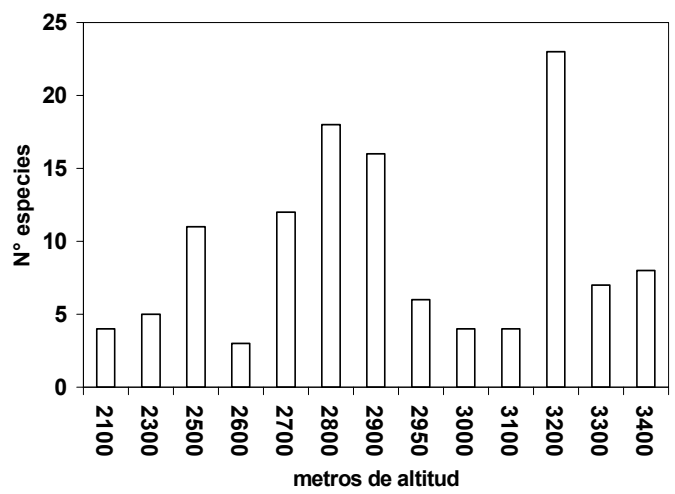

Figura 4. Número de especies endémicas encontradas (total de todas los sectores de estudio) en vegetación natural de acuerdo al gradiente de altitud.

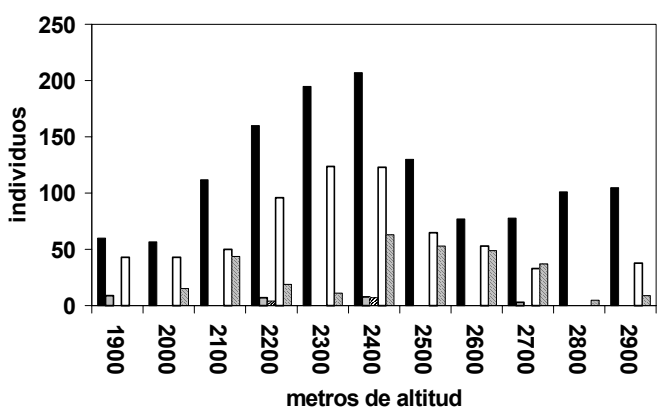

herbal $\square$ high herbal $\mathbb{2}$ high shrub $\square$ medium herbal $\square$ shrub

Figura 5. Distribución de estratos en deslizamientos naturales así como antrópicos según la gradiente altitudinal.

especiación y agrupamiento de las endémicas. Elementos como nitrógeno, materia orgánica, altitud y fisiografía, se muestran predominantes para el ordenamiento de la vegetación y cambian a lo largo de la gradiente, resultando relevante para la composición florística, el mayor endemismo se agrupa en el rango altitudinal 2700 a $2900 \mathrm{~m}$, con un pico alto en los $3200 \mathrm{~m}$ que refleja la diversidad de Cerro Toledo (Fig. 4).

La vegetación pionera que ocurre en los deslizamientos de los ex bosques montanos se caracteriza de acuerdo a los estratos principalmente, el más representativo es el estrato herbáceo bajo, seguido de un estrato herbáceo de tamaño mediano y finalmente el estrato arbustivo, especialmente en los rangos 2400 a $2700 \mathrm{~m}$, obviamente este factor lo determina la edad del deslizamiento, factor no fácil de definir debido a la constancia de los deslizamientos en la zona (Fig. 5).

La diversidad general encontrada en deslizamientos naturales y antropogénicos, comparada con la vegetación de bosque se representó de la siguiente manera:

D. Naturales

Familias Géneros Especies

D.Antropogénicos $69 \quad 152 \quad 313$

Bosque natural $\quad 75 \quad 185 \quad 412$ 


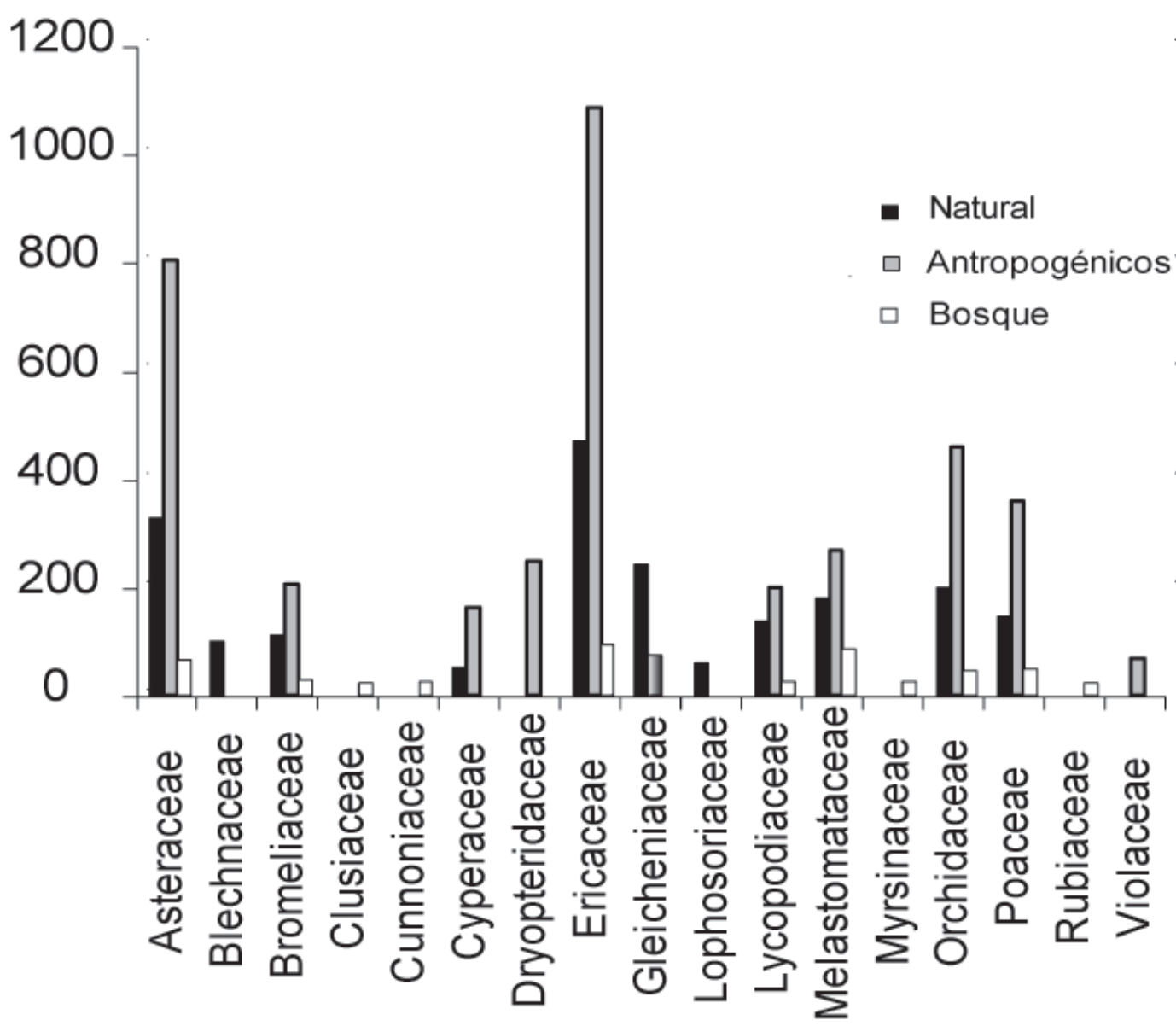

Figura 6. Suma acumulativa de todos los individuos presentes en las familias dominantes según los tipos de tratamiento: derrumbes naturales, antrópicos y vegetación de bosque natural.

Obviamente la diversidad existente en los bosques supera el de las áreas abiertas además de ser la que abastece de recursos genéticos para la repoblación de éstas. Se reconoce un mayor número de especies en los deslizamientos antropogénicos debido a cierta «estabilidad» que mantienen los taludes de carretera donde la vegetación se ha desarrollado de mejor manera, a diferencia de los deslizamientos naturales donde no se encontraron estados avanzados de la vegetación pionera, las familias dominantes son diferentes para cada tipo de perturbación y bosque. En las Figuras 6 y 7 , se presentan las familias y géneros más representativos para los tres tipos de análisis.

Estos datos preliminares encuentran que en los flancos del Parque Podocarpus existe una elevada frecuencia de deslizamientos prin- cipalmente entre los 2200-2500 m. La inclinación sumada al poco suelo favorece su presencia. Existen algunos grupos predominantes o que restablecen estas áreas, entre ellas las Criptógamas que están presentes en toda la gradiente, seguido de los estratos herbáceos con familias dominantes como (Violaceae, Cyperaceae, Poaceae, Blechnaceae, Lycopodiaceae), el estrato arbustivo con familias como (Asteraceae, Melastomataceae, Ericaceae, Poaceae, Aquifoliaceae), mientras que en el Bosque las familias dominantes son (Asteraceae, Clusiaceae, Bromeliaceae, Ericaceae, Lycopodiaceae, Melastomataceae, Orchidaceae, Poaceae y Cunnoniaceae).

Factores como pendiente, materia orgánica, acidez y estructura cambian en la gradiente e influyen directamente en la composición de 


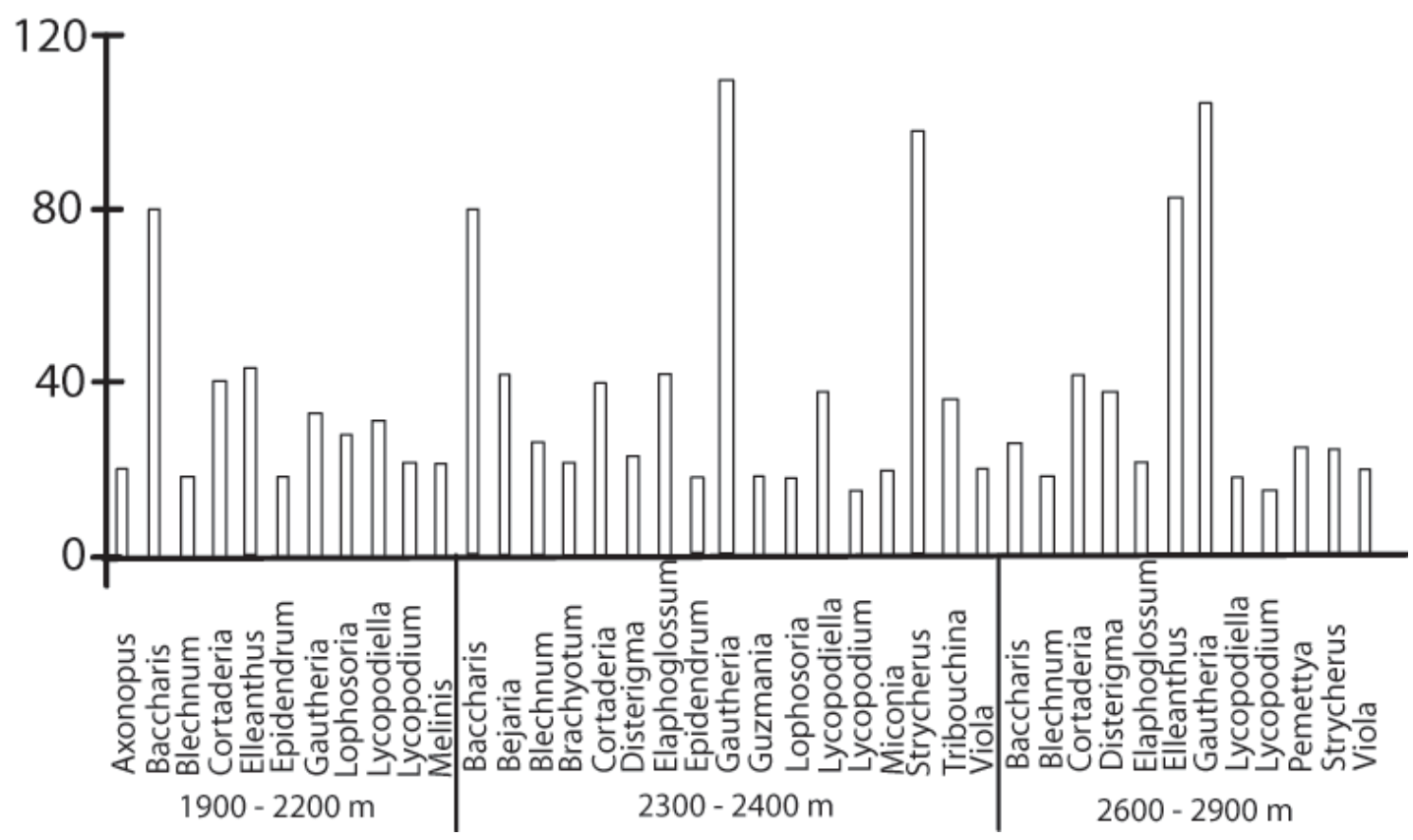

Figura 7. El mayor número de individuos por principales géneros encontrados según piso altitudinal (gradiente en deslizamientos naturales y antropogénicos).

la flora, análisis multivariados que involucran tanto especies con variables ambientales denotan estos factores como principales en la composición y agrupación de la flora. La dinámica de regeneración, así como las interacciones planta-animal que ocurren en estos procesos son numerosos e incluirían diversos tópicos de estudios, estos «gaps» constituyen un motor que genera una nueva fase de vida en el bosque.

\section{Literatura Citada}

Apolo, W. 1984. Plan de Manejo del Parque Nacional Podocarpus. Ministerio de Agricultura y Ganadería, Quito.

Benitez, A. 1989. Extend and Economic Significance in Ecuador. In: Landslides: Extent and Ecological Significance. Brabb \& Harrod (Eds.). Balkema, Rotterdam.

Bergeron, Y. \& Brisson, J. 1990. Fire regime in red pine stands at the northern limit of the species range. Ecology 71:1352-1364.

Bussmann, R. W. 2002. Estudio Fitosociológico de la Vegetación en la Reserva Biológica de San Francisco (ECSF), Zamora Chinchipe, Herbario Loja 8. Loja.
Bussmann, R. 2001. Epiphyte Diversity in a Tropical Andean Forest-Reserva Biológica San Francisco, Zamora-Chinchipe, Ecuador. Ecotropica 7: 43-59.

Brako, L. \& J. L. Zarucchi. 1993. Catalogue of the Flowering Plants and Gymnosperms of Peru. Syst. Bot. Mongr. 45. Missouri Botanical Garden. 1286 pp.

Braun Blanquet, J. 1979. Fito Sociología-Bases para el Estudio de las Comunidades Vegetales. Ediciones H.Blume. Madrid, España. 820 pp.

Cañadas-Cruz, L. 1983. El mapa bioclimático y ecológico del Ecuador. Quito, Ecuador, MAGPRONAREG. $210 \mathrm{p}$.

Christensen, L.J.. 1989. An Early Status of Regeneration in Gaps in a Montane Rain Forest in Southern Ecuador. Specialerapport. Botanisk Institut. Denmark. 76 pp.

Harling, G. \& L. Andersson (eds.). 1986-2003. Flora of Ecuador. 25-60.

Herbario Reinaldo Espinosa. 2000. Diagnostico de la vegetación Natural y de la intervención humana en los Páramos del PNP. Informe. Herbario Reinaldo Espinosa, Loja, Ecuador. 75 p.

Jørgensen, P.M. \& Leon-Yánez, S. 1999. Catalogue of the Vascular Plants of Ecuador. Ann. Missouri Botanical Garden. 75; 1-.1181. 
Keating, P. 1995. Disturbance Regimes and Regeneration Dynamics of Upper Montane Forest and Páramos in the Southern Ecuadorian Andes. Ph.D. thesis. Faculty of Geography, University of Colorado. 301 pp.

Lozano, P.; Delgado, T. Y Z. Aguirre M. 2003. Estado Actual de la Flora Endémica Exclusiva y su Distribución en el Occidente del Parque Nacional Podocarpus. Publicaciones de la Fundación Ecuatoriana para la Investigación y Desarrollo de la Botánica. Loja, Ecuador.

Madsen, J. E. 1989. Aspectos Generales de la Flora y Vegetación del Parque Nacional Podocarpus. En Boletín Informativo sobre la Biología, Conservación y Vida Silvestre. Pp 59-74. Universidad Nacional de Loja, Facultad de Ciencias Veterinarias. Loja Ecuador.

Ohl, C. \& Bussmann, R. 2004. Recolonisation of Natural Landslides in Tropical Mountains Forest of Southern Ecuador. Feddes Repertorium 115: 3-4; 248-264.

Peet \& Christensen. 1980. Succession: A Population Process. Vegetation. 43; 131-140.
Quizhpe, W.; Aguirre, Z.; Cabrera, O \& T. Delgado. 2002 Los Páramos del Parque Nacional Podocarpus. Pp. 79-89. en Z. Aguirre M., J.E. Madsen, E. Cotton y H. Balslev (eds.), Botánica Austroecuatoriana - Estudios sobre los Recursos Vegetales en las Provincias de El Oro, Loja y Zamora-Chinchipe. Ediciones Abya Yala, Quito.

Stern, M.J. 1992. Ecosystem Response to Natural and Anthropogenic Disturbances in the Andean Cloud Forest of Ecuador. Ph.D. Thesis. University of California.

Valencia, R.; Pitman, N.; León-Yánez, S. \& P. Jørgensen (eds.) 2000. Libro rojo de las plantas endémicas del Ecuador 2000. Herbario QCA, Pontificia Universidad Católica del Ecuador, Quito, Ecuador. Pp. 1-489.

White \& Jentsch. 2001. The Search for Generality in Studies of Disturbance and Ecosystem Dynamics. Progress in Botany 62: 399-449 Springer, Berlin Heidelberg.

Young, K. 1994. Roads and Environmental Degradation of Tropical Montane Forest. Conservation Biology. 8: 4; 972-976. 